\title{
Bound states solution of Klein-Gordon Equation with type - I equal vector and Scalar Poschl-Teller potential for Arbitray $l-$ State
}

\author{
${ }^{*}$ Akpan N. Ikot, ${ }^{2}$ Akpan B. Udoimuk and ${ }^{1}$ Louis E. Akpabio \\ ${ }^{1}$ Theoretical Physics Group, Department of Physics, University of Uyo, Nigeria. \\ ${ }^{2}$ Department of Physics, University of Calabar, Nigeria. \\ *Corresponding author, e-mail: ndemikot2005@yahoo.com
}

\begin{abstract}
We solve the bound state solution of Klein-Gordon equation for Poschl-Teller potential via the Nikiforov-Uvarov method. We obtain the energy eigen values and the wave function in terms of hypergeometric function.
\end{abstract}

Keywords: Klein-Gordon Equation, equal vector,

\section{INTRODUCTION}

In theoretical physics, one of the interesting problems is to obtain an exact solution of the Schrödinger, Klein-Gordon, Duffin-Kemmer-Petain and Dirac equation for mixed Vector and Scalar potentials [1-3]. These equations are frequently used to describe the particle dynamics in non-relativistic and relativistic quantum mechanics. However, the searches for the exact solutions of these equations have an important research area since the birth of quantum mechanics [4-5].

Consequently, the bound state solutions of KleinGordon equation and Dirac equation with mixed Vector and scalar potentials have significantly enriched ones knowledge of atomic and sub-atomic systems [6-7]. In recent times, a great attention has been spent to solve these non-relativistic and relativistic wave equations for various potentials using different methods [8-11]. Relativistic equations contain two objects: the four-vector linear momentum operator and the scalar rest mass. These allows one to introduce two types of potential coupling which are the four vector potential $V(r)$ and the space time scalar potential $\mathrm{S}(\mathrm{r})$.

Many authors by using different methods have studied the bound states of the Klein-Gordon equation and the Dirac equation with mixed typical potential under the condition that each of the scalar potentials is equal to its vector potential $V(r)$ [12].

These investigations include the Hulthen potential [13-14], Morse potential [15-16]. Poschl-Teller potential [17-18], Kratzer potential [19-20], Rosen Morse type potentials [21], the Coulombic ringshaped potential [22-23]. The methods include the standard method [24], super-symmetry and the shape invariance potential [25] and the NikiforovUvarov method [26]. The purpose of the present paper is to solve the Klein-Gordon equation for equal scalar and Vector type-I Poschl-Teller potentials with arbitrary angular momentum quantum number using the Nikiforov-Uvarov method [26].

The organization of the paper is as follows. In section 2, we review the Nikiforov-Uvarov [NU] method. We present factorization method in section 3 . The exact solution of the Klein-Gordon equation is given in section 4. Finally, a brief conclusion is presented in section 5 .

\section{Review of Nikiforov-Uvarov Method}

The NU method is based on solving a second order linear differential equation by reducing it to a generalized equation of hypergeometric type [26]. This method has been used to solve the Schrödinger, Dirac and Klein-Gordon equation for different kind of potential [19-22]. In NU method, the second order differential equation can be written in the form.

$\psi^{\prime \prime}(s)+\frac{\bar{\tau}(s)}{\sigma(s)} \psi^{\prime}(s)+\frac{\bar{\sigma}(s)}{\sigma^{2}(s)} \varphi(s)=0$,

when $\sigma(s)$ and $\bar{\sigma}(s)$ are polynomials at most of second degree and $\bar{\tau}(s)$ is a first degree polynomial. We write the transformation for the wave function in equation (1) as

$\psi(s)=\varphi(s) \chi_{n}(s)$

and this reduces equation (1) to equation of hypergeometric type,

$\sigma(s) \chi_{n}^{\prime \prime}(s)+\tau(s) \chi_{n}^{\prime}(s)+\lambda \chi_{n}(s)=0$, 
$\frac{\varphi(s)}{\varphi(s)}=\frac{\pi(s)}{\sigma(s)}$

The other wave function $\chi_{n}(s)$ is the hyper geometric function whose solutions are obtained by the Rodriques relation [26]

$\chi_{n}(s)=\frac{B_{n}}{\rho(s)} \frac{d}{d s^{n}}[\sigma(s) \rho(s)]$,

where $\mathrm{B}_{\mathrm{n}}$ is a normalization constant and $\rho(s)$ is the weight function that must satisfy the condition

$\frac{d}{d s}(\sigma(s) \rho(s))=\tau(s) \rho(s)$

The function $\pi(s)$ and the parameter $\lambda$ required for the NU-method are defined as follows:

$$
\begin{aligned}
& \pi(s)=\frac{\sigma^{\prime}-\bar{\tau}}{2} \pm \sqrt{\left(\frac{\sigma^{\prime}-\bar{\sigma}}{2}\right)^{2}-\bar{\sigma}(s)+k \sigma(s)} \\
& \lambda=k+\pi^{\prime}(s)
\end{aligned}
$$

In order to find the $\mathrm{k}$-value, the expression under the square root must be the square of a polynomial. Thus, a new eigenvalue equation for the secondorder differential equation becomes,

$\lambda=\lambda_{n}=-\frac{n d \tau(s)}{d s}-\frac{n(n-1)}{2} \frac{d^{2} \sigma(s)}{d s^{2}}$,

$\tau(s)=\bar{\tau}(s)+2 \pi(s) \quad$,

and its derivative is negative. Thus, by the comparison of equation (8) and equation (6), we obtain the energy eigenvalues.

\section{Factorization Method}

In spherical co-ordinates, the Klein-Gordon equation for a particle in generalized type -I Poschl-Teller potentials, with vector potential $V(r)$ and Scalar potential $S(r)$ read [3],

$\left[\hbar^{2} c^{2} \nabla^{2}+(E-V(r))^{2}-\left(m_{0} c^{2}+S(r)\right)^{2} \mid \psi(r, \theta, \varphi)=0\right.$,

or explicitly, we write

$$
\begin{aligned}
& -\hbar^{2} c^{2}\left[\frac{1}{r^{2}} \frac{\partial}{\partial r}\left(r^{2} \frac{\partial}{\partial r}\right)+\frac{1}{r^{2} \operatorname{Sin} \theta} \frac{\partial}{\partial \theta}\left(\operatorname{Sin} \theta \frac{\partial}{\partial \theta}\right)\right. \\
& \left.+\frac{1}{r^{2} \operatorname{Sin}^{2} \theta} \frac{\partial}{\partial \varphi^{2}}\right] \psi(r, \theta, \varphi)-\left\{(E-V(r))^{2}-\left(m_{0} c^{2}+S(r)\right)^{2}\right\} \\
& \psi(r, \theta, \varphi)=0,
\end{aligned}
$$

where $\mathrm{E}$ is the energy spectrum and $m_{0}$ is the rest mass of the particle. In order to find an exact solution to equation (12), we give spherical total wave function as

$\psi(r, \theta, \varphi)=R(r) H(\theta) \phi(\varphi) \quad$,

Substituting equation (13) into Schrödinger equation (12), the wave equation separated into independent variable and the following equations are obtained,

$$
\begin{gathered}
{\left[\frac{1}{r^{2}} \frac{d}{d r}\left(r^{2} \frac{d}{d r}\right)-\frac{\lambda}{r^{2}}+\frac{(E-V(r))^{2}-\left(m_{0} c^{2}+S(r)\right)^{2}}{\hbar^{2} c^{2}}\right] R(r)=0} \\
\frac{d^{2}}{d \theta^{2}} H(\theta)+\operatorname{Cot} \theta \frac{d H(\theta)}{d \theta}+\left[\lambda-\frac{m_{l}^{2}}{\operatorname{Sin}^{2} \theta}\right] H(\theta)=0,
\end{gathered}
$$

and

$$
\frac{d^{2} \phi(\varphi)}{d \varphi^{2}}+m_{l}^{2} \phi(\varphi)=0,
$$

where $\lambda$ is the separation constant. The solutions of equations (15) and (16) are the spherical harmonics $Y_{l m}(\theta, \varphi)$ and are well known [1] with $\lambda=l(\ell+1)$, where $\ell$ is the orbital angular momentum $\ell=0,1,2 \ldots$ and the magnetic quantum number $m_{\ell}=0, \pm 1 \pm 2 \cdots \pm \ell$.

$u(r)=r R(r)$,

Now using an ansaltz,

for the radial wave function in equation (14) transform into

$\left[\frac{d^{2}}{d r^{2}}-\frac{\ell(\ell+1)}{r}+\frac{(E-V(r))^{2}-\left(m_{0} c^{2}+S(r)\right)^{2}}{\hbar^{2} c^{2}}\right] u(r)=0$

where $\frac{1}{r^{2}} \ell(\ell+1) \hbar c^{2}$ is the centrifugal potential and the natural unit $\hbar=c=1$ will be use hence with. In this paper, we demonstrate the application of the NUmethod for the type-I Poschl-Teller potentials. The equal vector and scalar type - 1 Poschl-Teller potential is given by [27],

$$
V(r)=S(r)=\frac{A}{\operatorname{Sin}^{2} \alpha r}+\frac{B}{\operatorname{Cos}^{2} \alpha r}
$$

The Centrifugal term together with the equal scalar and Vector Type - I Poschl-Teller potential give rise to the effective potential

$V_{\text {eff }}(r)=\frac{\ell(\ell+1)^{2}}{r^{2}}+\left(\frac{A}{\operatorname{Sin}^{2} \alpha r}+\frac{B}{\operatorname{Cos}^{2} \alpha r}\right) \alpha^{2} / m$

This effective potential cannot be solved analytically for $\ell \neq 0$ because of the centrifugal term [3].

However, in order to obtain the analytical solutions of equation (18) with scheme suggested by C. S. Jia et al [17] to deal with the centrifugal term. When $\alpha r<<$, we use a modified new improved approximation scheme to deal with the centrifugal term

$\frac{1}{r^{2}}=4 \alpha^{2}\left[C_{0}-\frac{1}{4 \operatorname{Sin}^{2} \alpha r}\right]$,

where the parameter $C_{0}=1 / 12$ is a dimensionless constant. Figure 1 shows the variation of Type -1 Poschl-Teller Potential with $r$ for S-State, P-State and D-State. 
Am. J. Sci. Ind. Res., 2011, 2(2): 179-183

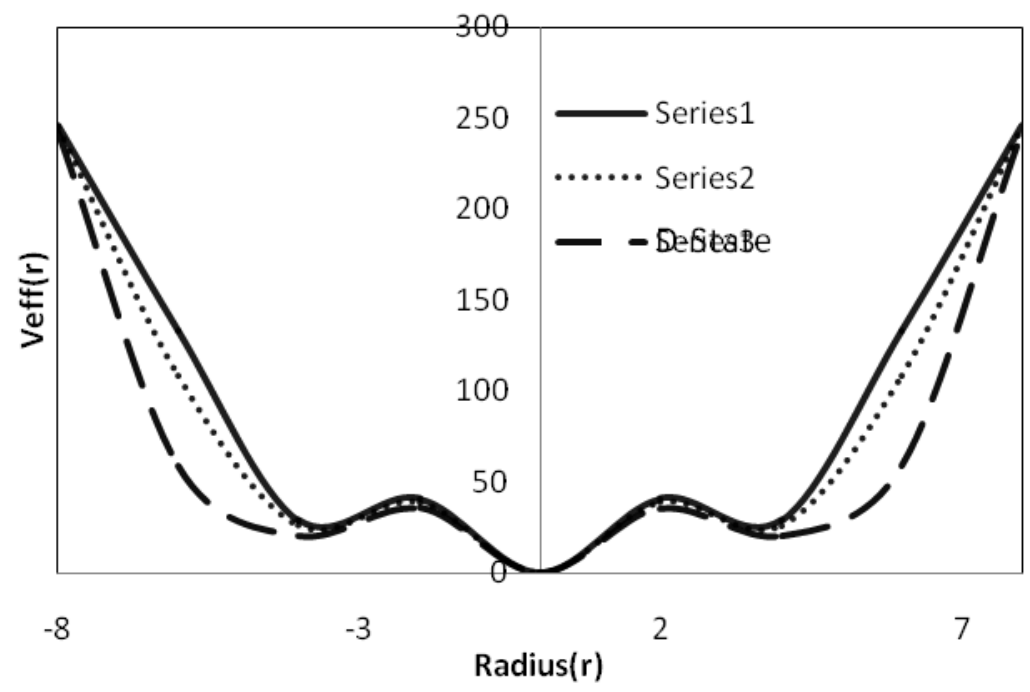

Fig.1: Variation of the effective potential as a function of $r$. The curves are shown for S-state, P-state and D-state.

\section{Bound State Solutions of Klein-Gordon Equation} In the presence of a Vector Potential $V(r)$ and $a$ Scalar Potential $S(r)$, the type - I Poschl - Teller Potential of equation (20), we write the Klein-Gordon equation as

$\left[\frac{d^{2}}{d r^{2}}-\ell(\ell+1)\left(C_{0}-\frac{1}{4 \operatorname{Sin}^{2} \alpha r}\right)-2\left(E+m_{0}\right)\left[\frac{A}{\operatorname{Sin}^{2} \alpha r}+\frac{B}{\operatorname{Cos}^{2} \alpha r}\right.\right.$

$\left.+\left(E^{2}-m_{0}^{2}\right)\right) u(r)=0$,

where $\mathrm{A}, \mathrm{B}$ and $\alpha$ are positive parameters.

In order to find the bound state solution of equation (22), we set

$S=\operatorname{Sin}^{2} \alpha r$,

and this transform equation (22) into

$$
\begin{aligned}
& S(1-s) \frac{d^{2} u}{d s^{2}}+\left(\frac{1}{2}-s\right) \frac{d u}{d s}-\left[\frac{\left(E+m_{0}\right) A}{2 \alpha^{2} s}+\frac{\left(E+m_{0}\right) B}{2 \alpha^{2}(1-s)}\right. \\
& \left.-\frac{\left(m_{0}^{2}-E^{2}\right)}{4 \alpha^{2}}-\frac{\ell(\ell+1) C_{0}}{4 \alpha^{2}}+\left(\frac{\ell(\ell+1)}{16 \alpha^{2}}\right)\right] u(r)=0
\end{aligned}
$$

Now setting the following dimensionless parameters:

$$
\begin{gathered}
\varepsilon^{2}=\left(m_{0}^{2}-E^{2}\right) / \alpha^{2} \quad, \quad \beta^{2}=2 \frac{\left(E+m_{0}\right)}{\alpha^{2}} A=2 \bar{V}_{0} / \alpha^{2} \\
\gamma^{2}=2 \frac{\left(E+m_{0}\right)}{\alpha^{2}} B=2 \bar{V}_{1} / \alpha^{2}, \quad \delta^{2}=\frac{\ell(l+1) C_{0}}{4 \alpha^{2}} \\
\wedge^{2}=\ell(\ell+1) / 4 \alpha^{2}
\end{gathered}
$$

turns equation (23) into a second order differential equation of the hypergeometric type.

$$
\begin{gathered}
\frac{d^{2} u}{d s^{2}}+\frac{1-2 s}{2 s(1-s)} \frac{d u}{d s}-\frac{1}{4 s^{2}(1-s)^{2}}\left[\left(\varepsilon^{2}+\delta^{2}\right) s^{2}\right. \\
+\left(r^{2}-\beta^{2}-\delta^{2}-\wedge^{2}-\varepsilon^{2}\right) s+\left(\beta^{2}+\wedge^{2}\right) u(r)=0
\end{gathered}
$$

If we apply the NU method in the present case by comparing equation (25) with equation (1), we obtained the following expressions:

$\bar{\tau}=1-2 s, \quad \sigma(s)=2 s(1-s)$

$\bar{\sigma}(s)=-a s^{2}+b s-c \quad$,

where

$$
\begin{gathered}
a=-\left(\varepsilon^{2}+\delta^{2}\right) \\
b=\left(\beta^{2}+\delta^{2}+\wedge^{2}+\varepsilon^{2}-r^{2}\right) \\
c=-\left(\beta^{2}+\wedge^{2}\right)
\end{gathered}
$$

Substituting equation (26) in to equation (7) and according to the property that $\pi(s)$ is a polynomial, we find $\pi(s)$ as

$$
\pi(s)=\frac{1-2 s}{2} \pm \frac{1}{2}\left\{\begin{array}{l}
\left(2 \sqrt{\xi_{1}}-\sqrt{\xi_{3}}\right) s-2 \sqrt{\xi_{1}} \\
\text { for } k=\frac{-\xi+2 \sqrt{\xi_{1} \xi_{3}}}{2} \\
\left(2 \sqrt{\xi_{1}}+\sqrt{\xi_{3}}\right) s-2 \sqrt{\xi_{1}}, \\
\text { for } k=\frac{-\xi_{2}-2 \sqrt{\xi_{1} \xi_{3}}}{2}
\end{array}\right.
$$

where

$$
\begin{aligned}
& \xi_{1}=a+4 \\
& \xi_{2}=b+4
\end{aligned}
$$

$\xi_{3}=c+1$

The suitable $\mathrm{k}$ in equation (28) is determined by the polynomial $\tau=\bar{\tau}+2 \pi$ having a negative derivative. Therefore, the polynomial $\pi(s)$ is computed from equation (28) as

$\pi(s)=\frac{1-2 s}{2}-\frac{1}{2}\left[\left(2 \sqrt{\xi_{1}}+\sqrt{\xi_{3}}\right) s-2 \sqrt{\xi_{1}}\right]$, 
and the function $\tau(s), \tau^{\prime}(s)$ satisfies these requirements as:

$\tau(s)=\frac{1-2 s}{2}-\left[\left(2 \sqrt{\xi_{1}}+\sqrt{\xi_{3}}\right) s-2 \sqrt{\xi_{1}}\right]$,

$\tau^{\prime}(s)=-1-\left(2 \sqrt{\xi_{1}}+\sqrt{\xi_{3}}\right)$

with this selection of $\tau(s), \pi(s), k$, and the

$\lambda=k+\pi^{\prime}(s)$, we obtain

$\lambda=-\xi_{2}-2 \sqrt{\xi_{1} \xi_{3}}-+-\sqrt{\xi_{1}}-\frac{1}{2} \sqrt{\xi_{3}}$,

Using the relation $\lambda_{n}=n \tau^{\prime}-n^{(n-1)} / 2 \sigma^{\prime \prime}$, we have

$\lambda_{n}=n+2 n \sqrt{\xi_{1}}+n \sqrt{\xi_{3}}+2 n(n-1)$,

Comparing eqns. (33) and (34), and using equations (24), and (29), we obtain the energy spectrum $\bar{E}$ as

$\bar{E}=\frac{-\alpha^{2}}{\left[1+\left(1+2 n+\sqrt{1+\frac{1}{4 \alpha^{2}}\left(8 \bar{v}_{1}+\ell(\ell+1)\right)}\right)^{2}\right]}\left[1+\frac{1}{2} \sqrt{1-\frac{1}{4 \alpha^{2}}\left(4 \bar{v}_{1}+\ell(\ell+1)\right.}\right.$

$\left.+\left(1+\sqrt{1-\frac{1}{4 \alpha^{2}}\left(4 \bar{v}_{1}+\ell(\ell+1)\right)}\right) n+2 n(n-1)-4\left(1-\frac{\bar{V}_{2}}{4 \alpha^{2}}-\frac{\bar{V}_{2}}{4 \alpha^{2}}+\ell(\ell+1)\right)\right]$

$\left\{1 \pm \sqrt{1+\left(1+2 n+\sqrt{1+\frac{1}{4 \alpha^{2}}\left(8 \bar{V}_{1}+\ell(\ell+1)\right)}\right)^{2}}\right\}+\frac{\ell(\ell+\ell) C_{0}}{4}$

Here the index $\mathrm{n}$ is a non-negative integers and equation (35) shows that we are dealing with a family of Posehl-Tellor Potential.

In this work, we study the bound state solutions, that is the radial equation of the wave function $u(r)$ that must satisfy the boundary condition $u(r) / r \rightarrow 0$ as $r \rightarrow 0$ and $u(r) / r$ become finite at $r=0$. In order to find the wave function $u(r)$, we consider equation (23), which has three singularities at $s=0,1$ and $\infty$, we make an ansaltz for the wave function as $u(s)=s^{(\varepsilon+\delta)}(1-s)^{\beta} f(s)$,

and $u^{\prime}(s)$ and $u^{\prime \prime}(s)$ becomes

$$
\begin{aligned}
& u^{\prime}(s)=\left[\frac{(\varepsilon+\delta)}{s} f(s)-\frac{\beta f(s)}{(1-s)}+f^{\prime}(s)\right] s^{\varepsilon+\delta}(1-s)^{\beta} \\
& u^{\prime \prime}(s)=\left[\frac{(\varepsilon+\delta)(\varepsilon+\delta-1)}{s^{2}} f(s)-\frac{2 \beta(\varepsilon+\delta) f(s))}{s(1-s)}+\frac{2(\varepsilon+\delta) f^{\prime}(s)}{s}\right. \\
&\left.-\frac{2 \beta f^{\prime}(s)}{1-s}+\frac{\beta(\beta-1) f(s)}{(1-s)^{2}}+f^{\prime \prime}(s)\right] s^{\varepsilon+\delta}(1-s)^{\beta},
\end{aligned}
$$

Substituting equations (35)-(37) into equation (23), we obtain.

$$
\begin{aligned}
& s(1-s) f^{\prime \prime}(s)+[1+4(\varepsilon+\delta)-4((\varepsilon+\delta)+\beta+1) s] f^{\prime}(s) \\
& -\left[(\varepsilon+\delta)^{2}+\beta^{2}+\varepsilon+\delta+\beta+2(\varepsilon+\delta) \beta+\gamma^{2}+\wedge^{2}\right] f(s)=0
\end{aligned}
$$

This equation is the well-known differential equation satisfied by the hypergeometric function ${ }_{2} F_{1}(a, b ; c, s)$, thus,

$$
f(s)=\frac{\Gamma(c)}{\Gamma(a) \Gamma(b)} \sum_{k=0}^{\infty} \frac{\Gamma(a+k) \Gamma(b+k)}{\Gamma(c+k)} s^{k} / k !
$$

\section{Where}

$$
\begin{aligned}
& a+b=2+(\varepsilon+\delta)+2 \beta+1 \\
& a b=(\varepsilon+\delta)^{2}+\beta^{2}+\varepsilon+\delta+\beta+2(\varepsilon+\delta) \beta+\gamma^{2}+\wedge^{2}, \\
& c=1+4(\varepsilon+\delta) .
\end{aligned}
$$

In terms of the variable $r$, we write the unnormalized radial wave function for the type I Poschl-Teller potential as

$R_{n l}(r)=\frac{1}{r} \sin ^{2(\varepsilon+\delta)}(\alpha r)\left(1-\sin ^{2(\xi+\delta)}\right)(\alpha r) \times 2 F_{1}(\xi, \rho, c)$,

Where $\xi=(a+b)$ and $\rho=a b$.

\section{CONCLUSION:}

We have investigated the bound state solution of the klein-Gordan with equal scalar and vector type I Poschl Teller potential for arbitrary angular momentum number $l$. We use the Nikiforov-Uvarov method to find exact the energy spectrum and the unnormalized wave function is obtained via an ansaltz and expressed in terms of the hypergeometric function. Finally, our results are consistence with that of ref.[3] using analytical methods.

\section{ACKNOWLEDGEMENT}

This work was supported partially by the NANDYLEABIO Foundation under grant No. 3726-421.

\section{REFERENCES}

1. S. Flugge, Practical Quantum Mechanics, Vol. 1

(35) (Springer, Berlin, 1994).

2. M. Simsek and H. Egrifes, J. Phys. A. Math Gen. 37, 4379 (2004).

3. Y. Xu, S. He and C. S. Jia, Phys. Scr. 81, 045001 (2010).

4. M. Kocak and B. Goniil, Clin Phys. Lett. 24, 3024 (2007)

5. B. Goniil, K. Koksal and E. Bakur, Phys. Scr. 73, 279, (2006).

6. L. Hulthen, Ark. Mat. Astran. Fus. 28A, 5 (1942)

7. V. P. Varshni, Phys. Rev. A 41, 4682 (1990) 
8. H. Egrifes, D. Demirhan and F. Buyukkhe, Physisca. Scripta, 59, 90 (1999)

9. H. Egrifes D. Demirhan and F. Buyukkhe, Physica Scripta, 60, 195 (1999)

10. M. Simek and H. Egrifes, J. Phas. A: Math. Gen. 37, 4379 (2004)

11. A. A. D Alhairlari, H. Bahlouli and A. Al-Hasan, Phys. Lett. A 349, 87 (2006)

12. P. Alberto, A. S. decastro and M. Malheiro, Phys. Rev. C. $25,047303,(2007)$

13. N. Saad, phys. Scr. 76, 623 (2007)

14. A. Arda and R. Sever, Int. J. Theor. Phys. 48, 945, (2009)

15 L. H. Zhang, X. P. Li and C. S. Jia, Phys. Scr. 80 035003 (2009)

16. N. Rosen and P. M. Morse, Phys. Rev. 42, 210 (1932)

17. C. S. Jia, T. Chen and L. G. Cui, Phys. Lett. A 373, 1621 (2009)
18. T. Chen, Y. F. Diao and C. S. Jia, Phys. Scr. 79, 065014, (2009)

19. J. Sadeghi and B. Pourhassan, EJTP, 5, 193 (2008)

20. Y. F. Cheng, and T. G. Dai, Phys. Scr. 75, 274 (2007)

21. A. N. Ikot, and L. E. Akpabio,accepted in Applied Physics Research(APR).

22. O. Yesiltas and R. Sevar, arxiv: quant-ph/0703034

23. B. M. Mandal, Int. J. Mod. Phys. A15, 1225 (2000)

24. W. Greiner, (Relativistic Quantum Mechanics,, Berlin, Springer, 1990)

25. F. Cooper, A. Khare and V. Sukhatme, Phys. Rep. 251, 267 (1995)

26. A. F. Nikiforov and V. B. Uvarov, (Special Functions of Mathematical Physics, Birkhaiiser, Basel, 1988)

27. G. Poschl and E. Teller, Z. Phys, 83, 143 (1933) 